\title{
COMMON ACRONYMS
}

AACC

ABET

ACM

ACM-W

ADMI

AIS

AISES

CPSR

CRA

American Association of Community Colleges https://www.aacc.nche.edu/

Accreditation Board for Engineering and Technology http://www.abet.org/

Association for Computing Machinery https://www.acm.org/

ACM Council on Women in Computing https://women.acm.org/

Association of Computer/Information Sciences and Engineering Departments at Minority Institutions https://www.admiusa.org/

Association for Information Systems https://aisnet.org/

The American Indian Science and Engineering Society http://www.aises.org/

Computer Professionals for Social Responsibility http://cpsr.org/

Computer Research Association https://cra.org/

EngageCSEdu Engage CS EDU https://www.engage-csedu.org/

HBCU

Historically Black Colleges and Universities https://www.niche.com/blog/list-of-hbcu-schools-in-america-2/

IAAMCS

IEEE

MAA

Institute for African-American Mentoring in Computing Sciences ('i am cs') http://www.iaamcs.org/

Institute of Electrical and Electronics Engineers https://www.ieee.org/index.htm/

Mathematical Association of America https://www.maa.org/

MAES Latinos in Science and Engineering http://mymaes.org/

NCWIT National Center for Women and Technology https://dev.ncwit.org/

NSBE National Society of Black Engineers http://www.nsbe.org/home.aspx

SHPE Society of Professional Hispanic Engineers http://www.shpe.org/

WICS

Women in Computer Science

https://www.computerscience.org/resources/women-in-computer-science/

\section{ACM Special Interest Groups}

$\begin{array}{ll}\text { SIGACCESS } & \text { Accessibility and Computing } \\ \text { SIGCAS } & \text { Computers and Society } \\ \text { SIGCHI } & \text { Computer-Human Interaction } \\ \text { SIGCSE } & \text { Computer Science Education } \\ \text { SIGGRAPH } & \text { Computer Graphics } \\ \text { SIGHPC } & \text { High Performance Computing } \\ \text { SIGITE } & \text { Information Technology Education } \\ \text { SIGMIS } & \text { Management Informaton Systems } \\ \text { SIGPLAN } & \text { Programming Languages } \\ \text { SIGSOFT } & \text { Software Engineering }\end{array}$

http://www.sigaccess.org/

http://www.sigcas.org/

http://www.sigchi.org/

http://www.sigcse.org/

http://www.siggraph.org/

http://www.sighpc.org/

http://www.sigite.org/

http://www.sigmis.org/

http://www.sigplan.org/

https://www.sigsoft.org/index.htm/ 